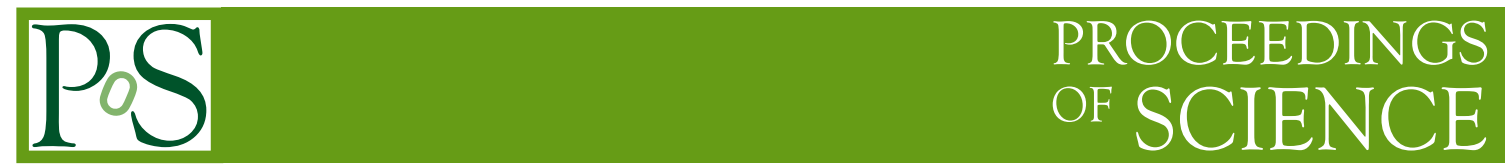

\title{
Potential of FORM 4.0
}

\author{
J.A.M.Vermaseren* \\ Nikhef, Science park 105, 1098XG, Amsterdam, The Netherlands \\ E-mail: t68@nikhef.nl
}

I describe the main new features of FORM version 4.0. They include factorization, polynomial arithmetic, new special functions, systems independent sav files, a complete ParForm, open source code and a forum for user communication.

Loops and Legs in Quantum Field Theory - 11th DESY Workshop on Elementary Particle Physics, April 15-20, 2012

Wernigerode, Germany

* Speaker. 


\section{Introduction}

Over the past 12 years FORM was available as version 3.0-3.3 [1, 2]. This version was significantly more powerful than version 2. But also these versions needed extensions. More recently there were a few opportunities to hire extra people for special contributions and development picked up speed. A number of much needed but very labor intensive projects were undertaken and completed. This marked a good point to clean up the whole program, make it open source and bring it out as a new version.

On March 29 version 4.0 was released. It took much more time than anticipated to prepare this release, because there are quite a few new features and the debugging was a slow procedure. In addition making the source code available and easy to install took a lot of work.

Most work for version 4.0 has been put in by Jan Kuipers, Takahiro Ueda, Jens Vollinga and me. Other people (and Jens) who worked on Form in the past have left the field and the current team consists of Jan Kuipers, Takahiro Ueda and me. Jan will leave in the autumn. This means that the speed of advances will be slowed down until there are new opportunities to hire good people.

Jens Vollinga has added a number of very nice features to FORM , including systems independent .sav files, checkpoints (points from which a program can be restarted if it crashes), much documentation, the forum and he designed much of the open source infrastructure.

Jan Kuipers has made the factorization and polynomial libraries and is currently working on a completely new method for output simplification[7, 8].

Takahiro Ueda has been hired by Karlsruhe on DFG money with the task to improve the parallelization of FORM . The project is to combine TFORM and ParFORM to make use of clusters of multicore computers. This project is still in its infancy, because his first task was to make ParForm complete. This has been finished now. He has also taken over the task to manage the open source infrastructure.

This talk will take us through a number of new features and in the end give a few hints about what the future might bring.

The first thing the user will notice is the new header.

FORM 4.0 (Mar 29 2012) 64-bits

Run: Wed Apr 4 14:23:50 2012

The mentioning of the 64/32-bits version is for version 4, because we are in a period that many people still use 32-bits computers or operating systems. Other headers are

TFORM 4.0 (Mar 29 2012) 64-bits 8 workers

Run: Wed Apr 4 14:24:22 2012

or

ParFORM 4.0 (Mar 29 2012) 64-bits 8 workers

Run: Wed Apr 4 14:32:45 2012

\section{Factorization}

The first feature we are going to look at is one that many people have asked for in the past. 
It should be realized that factorization is a subject that many mathematicians have given attention to. In addition big commercial programs have spent much effort on making good packages for this. Hence one should not expect to outperform other packages. The best model here is to look whether there are packages under the GNU license that have been created and are maintained by good mathematicians. Unfortunately we could not find any that deal with more than a single variable. The better packages are all closed source and part of a commercial system. This means we had to make our own. But then we could optimize it for what we anticipate that the use will be.

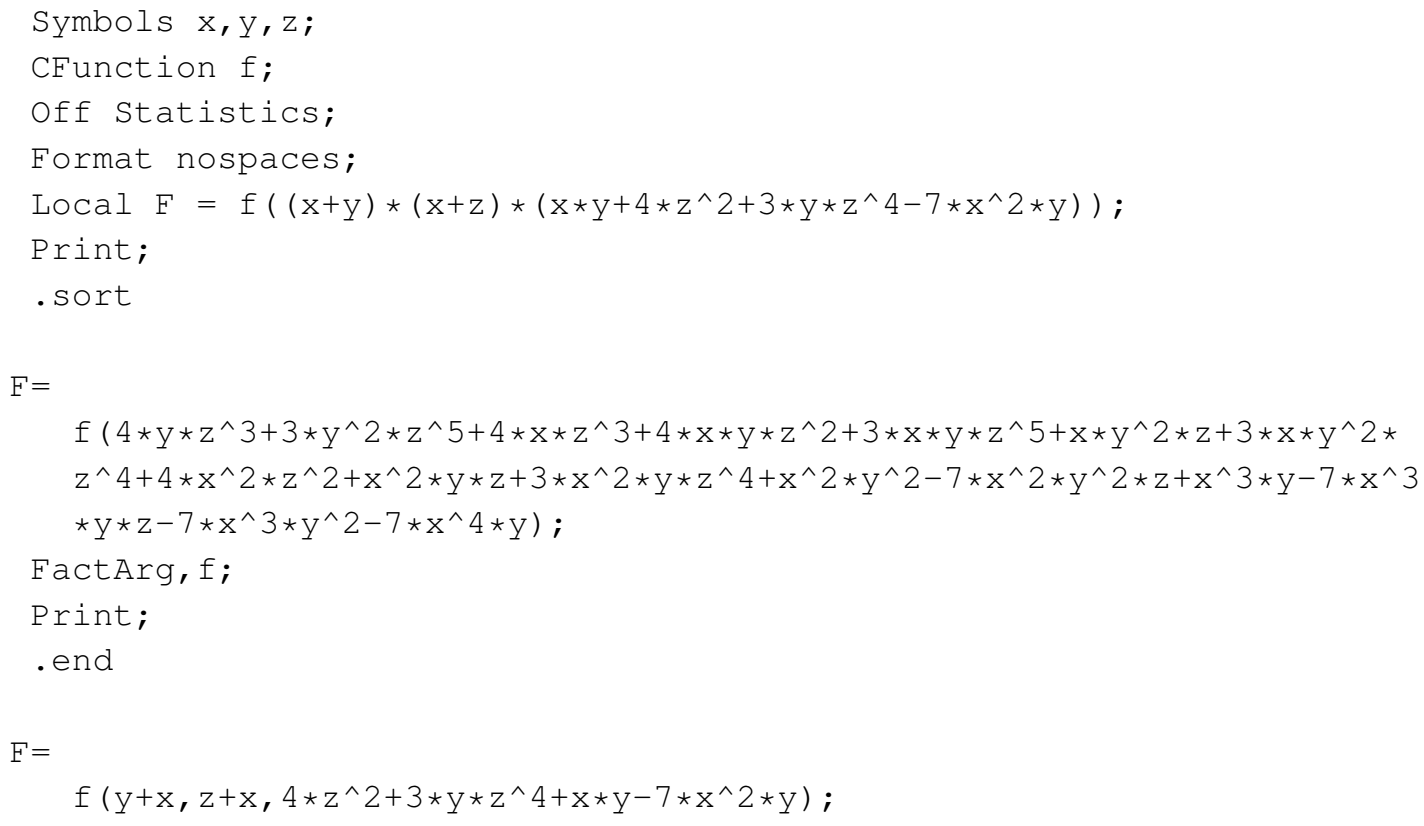

The first example shows the factorization of function arguments. This is probably the most important use in complicated calculations. The FactArg statement is natural for this.

Factorization of expressions is a bit more complicated. How to (re)present the results?

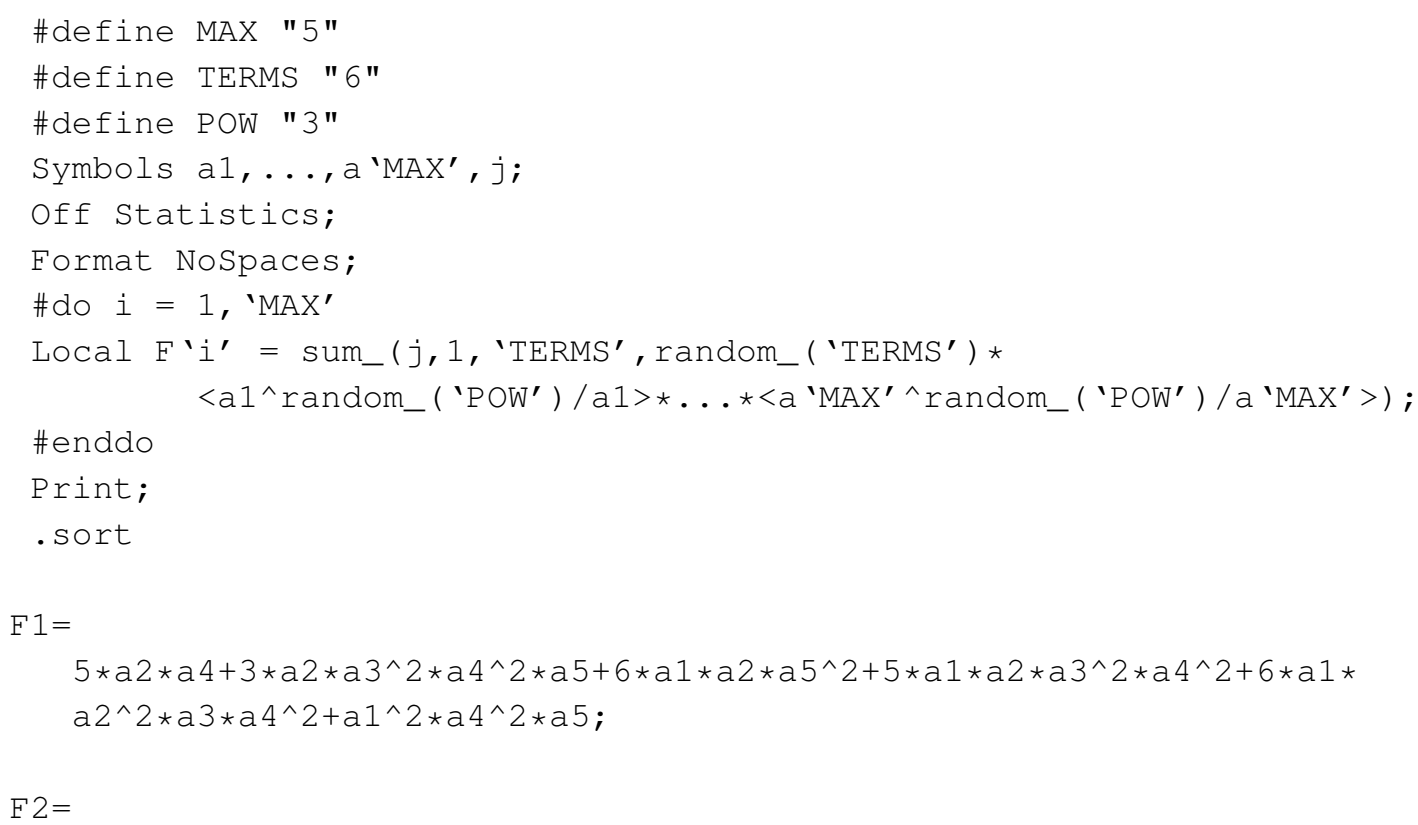


$2 * a 3^{\wedge} 2 * a 4^{\wedge} 2 * a 5+2 * a 1 * a 5+2 * a 1^{\wedge} 2 * a 3^{\wedge} 2 * a 4^{\wedge} 2+2 * a 1^{\wedge} 2 * a 2^{\wedge} 2 * a 5^{\wedge} 2+4 * a 1^{\wedge} 2 *$ $a 2^{\wedge} 2 * a 3 * a 4^{\wedge} 2 * a 5^{\wedge} 2+a 1^{\wedge} 2 * a 2^{\wedge} 2 * a 3^{\wedge} 2 * a 4 * a 5^{\wedge} 2$

$\mathrm{F} 3=$

$6 * a 3^{\wedge} 2 * a 4 * a 5^{\wedge} 2+5 * a 2 * a 3 * a 4^{\wedge} 2+a 1 * a 2 * a 3^{\wedge} 2 * a 5+a 1^{\wedge} 2 * a 3^{\wedge} 2 * a 4^{\wedge} 2 * a 5+4 *$ $a 1^{\wedge} 2 * a 2 * a 3 * a 4^{\wedge} 2+a 1^{\wedge} 2 * a 2^{\wedge} 2 * a 3^{\wedge} 2 * a 4^{\wedge} 2$

$\mathrm{F} 4=$

$6 * a 2^{\wedge} 2 * a 4^{\wedge} 2 * a 5+4 * a 2^{\wedge} 2 * a 3^{\wedge} 2 * a 4 * a 5^{\wedge} 2+4 * a 1 * a 2 * a 3^{\wedge} 2 * a 4^{\wedge} 2+a 1^{\wedge} 2 * a 2 * a 3+6$ $\star a 1^{\wedge} 2 * a 2 * a 3^{\wedge} 2 * a 5^{\wedge} 2+a 1^{\wedge} 2 * a 2^{\wedge} 2 * a 5^{\wedge} 2 ;$

$\mathrm{F} 5=$

$5 * a 2 * a 3^{\wedge} 2 * a 4^{\wedge} 2 * a 5+3 * a 1 * a 3^{\wedge} 2 * a 4^{\wedge} 2+2 * a 1 * a 2 * a 3 * a 4^{\wedge} 2 * a 5+a 1^{\wedge} 2 * a 3+5 *$

$a 1^{\wedge} 2 * a 2^{\wedge} 2 * a 3^{\wedge} 2 * a 4 * a 5+a 1^{\wedge} 2 * a 2^{\wedge} 2 * a 3^{\wedge} 2 * a 4 * a 5^{\wedge} 2$

On Statistics;

Drop;

Local $\mathrm{F}=\mathrm{F} 1 * \ldots \star \mathrm{F}^{\prime} \mathrm{MAX}^{\prime}$;

. sort

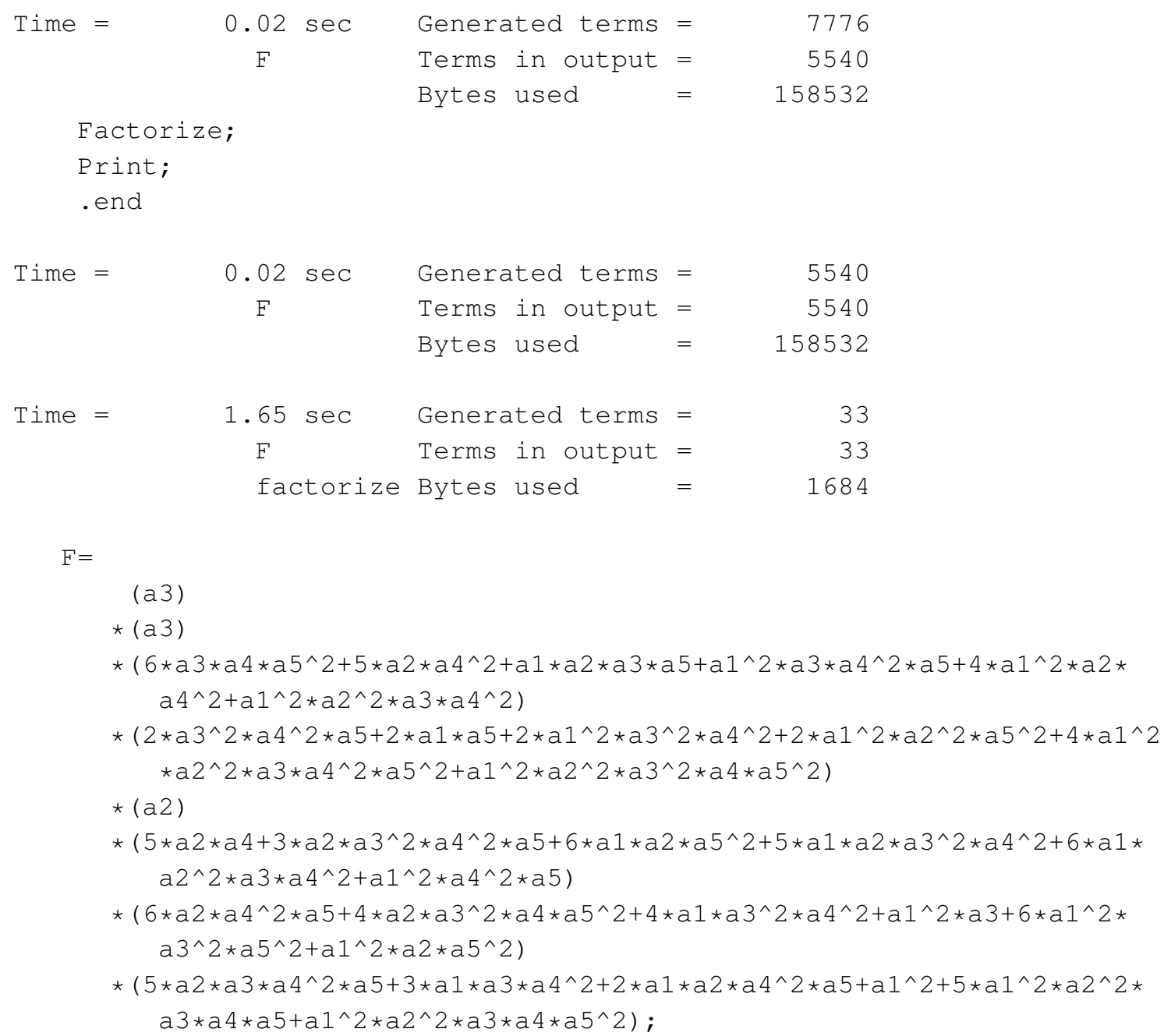

Factorization is considered a 'state' in which the expression exists. It is either factorized or 
unfactorized. Conversion takes place at the end of the module after the expression has been processed and sorted. Hence we have two output statistics. The second one refers to the factorization procedure. To store the factorized expression we use the FORM bracket system with the built in symbol factor. This allows also a way to refer to the brackets.

The execution time depends critically on how complicated the expression is. If we raise the powers of the variables we can see the effect:

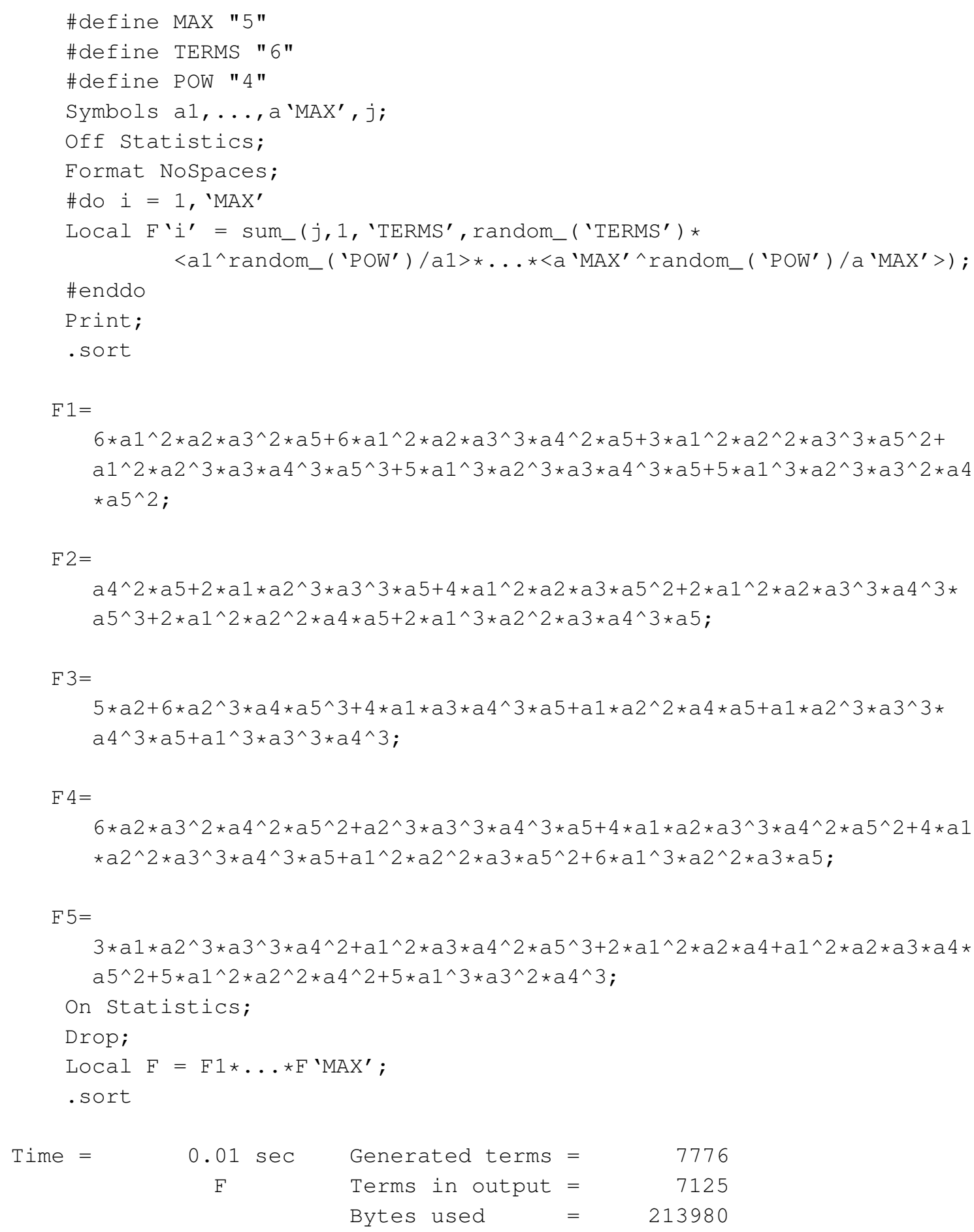

Bytes used $\quad=\quad 213980$




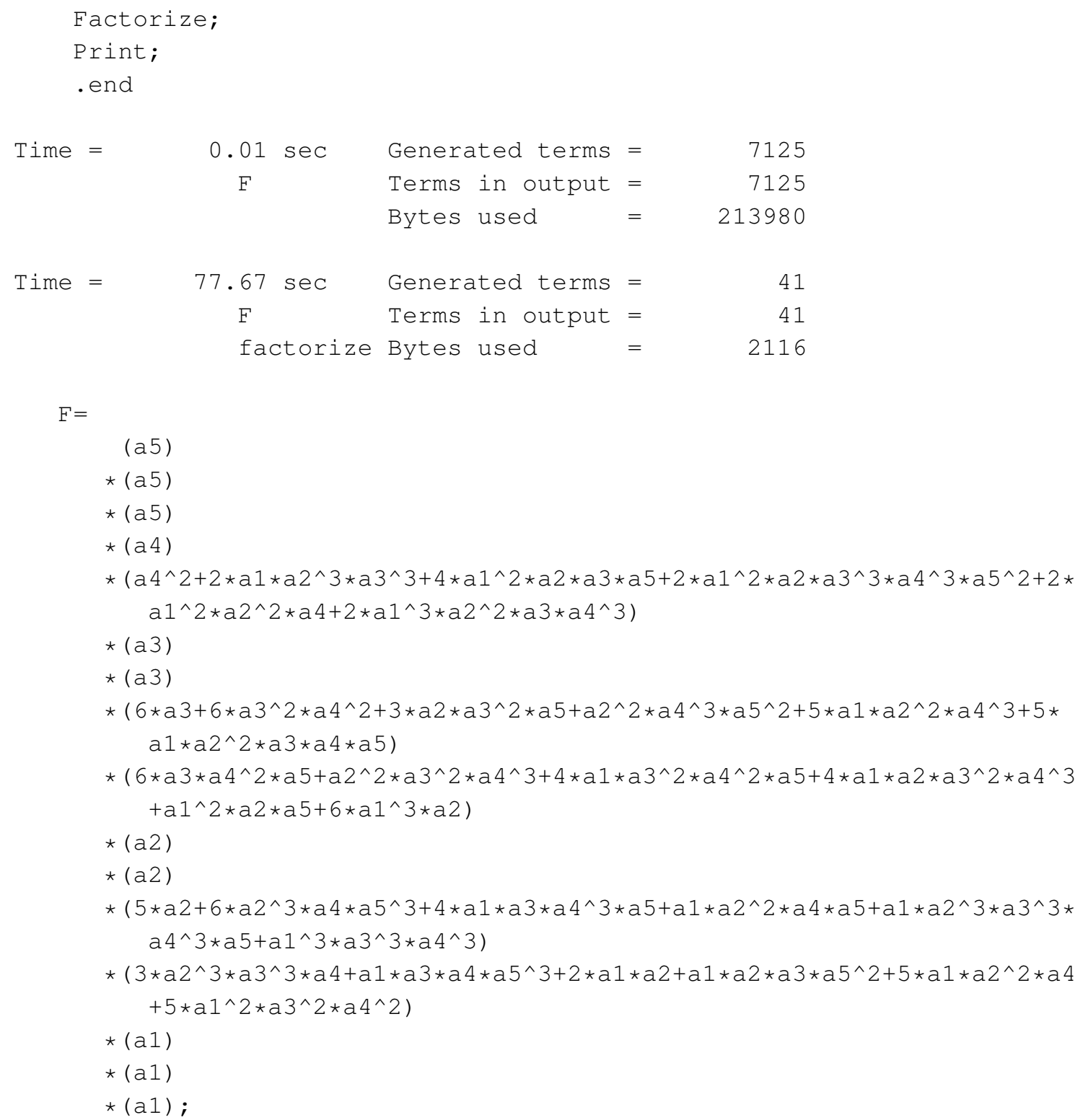

It is also possible to put expressions in the input in factorized form:

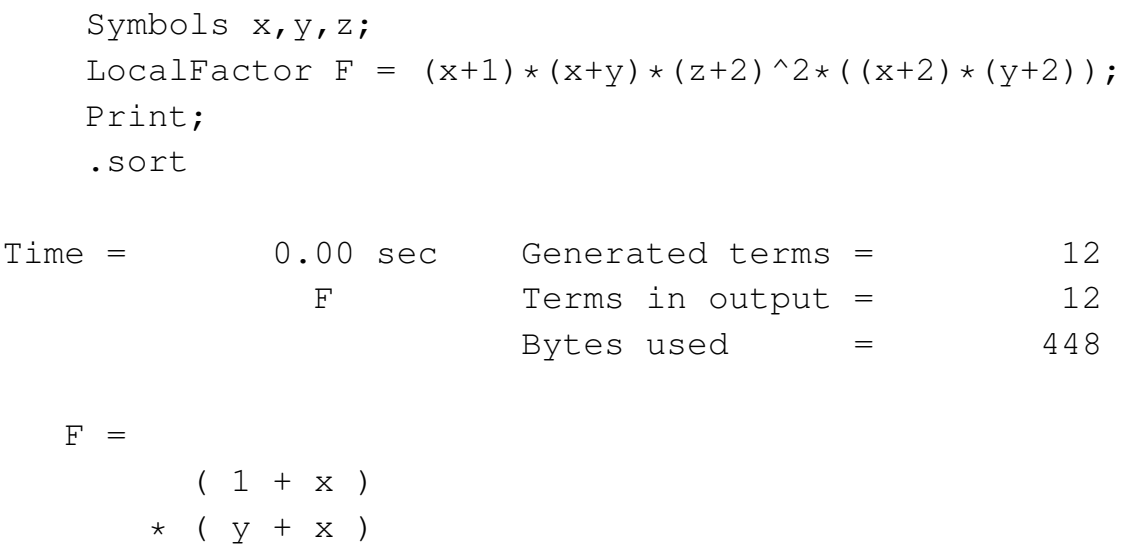




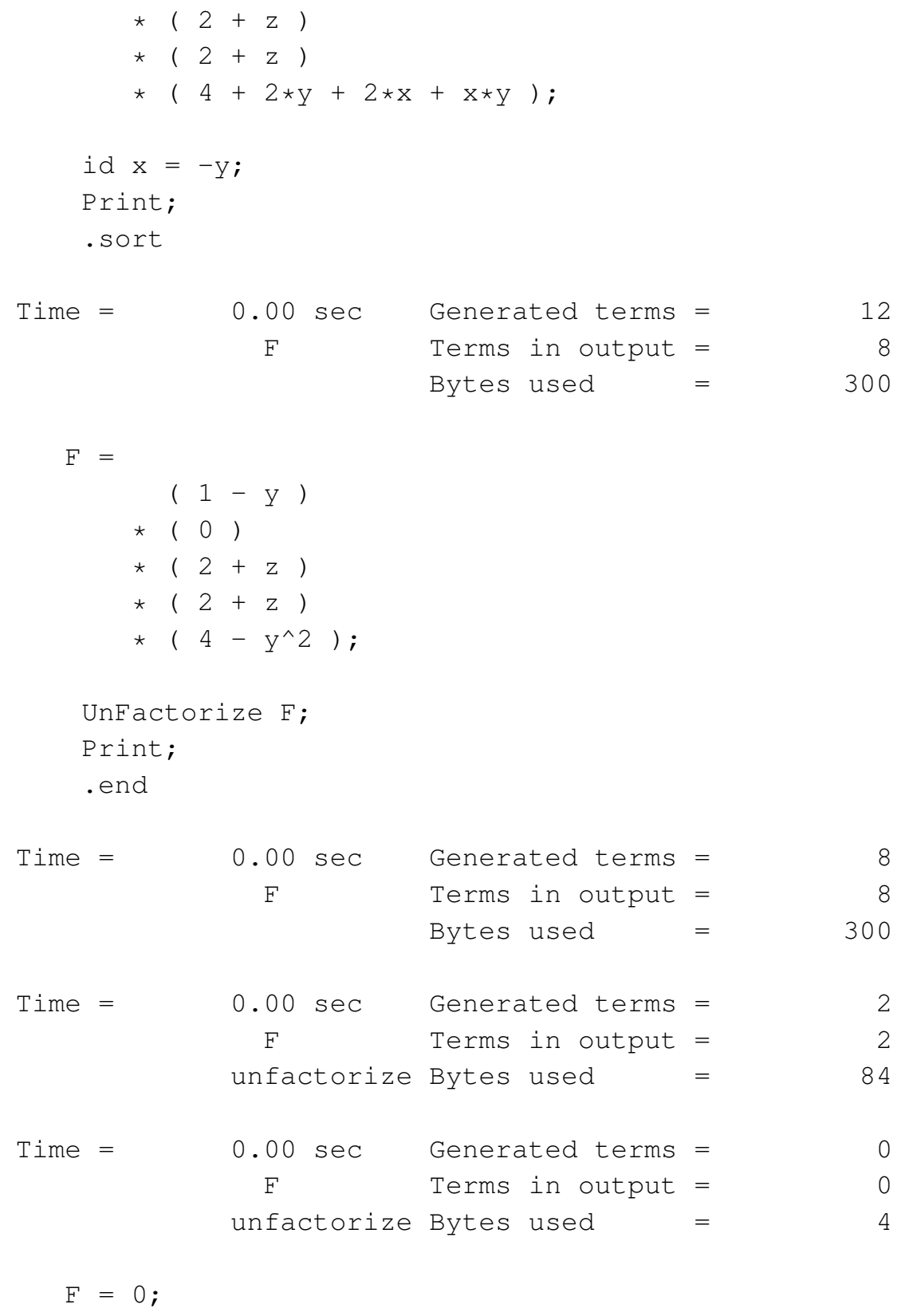

$\mathrm{F}=0$;

This example shows also that if during further processing a factor becomes zero, we still keep the expression and the other factors. If, on the other hand, we unfactorize the expression, we end up with zero of course.

Factorization of $\$$-expressions is yet another case. Here we do not have the bracket system. Neither do we have the possibility to store the factors as arguments. On the other hand, we are not limited by the maximum size of terms.

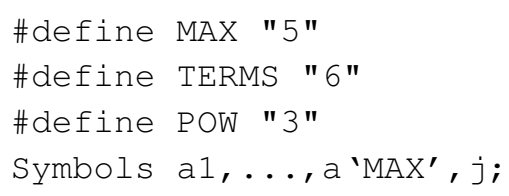




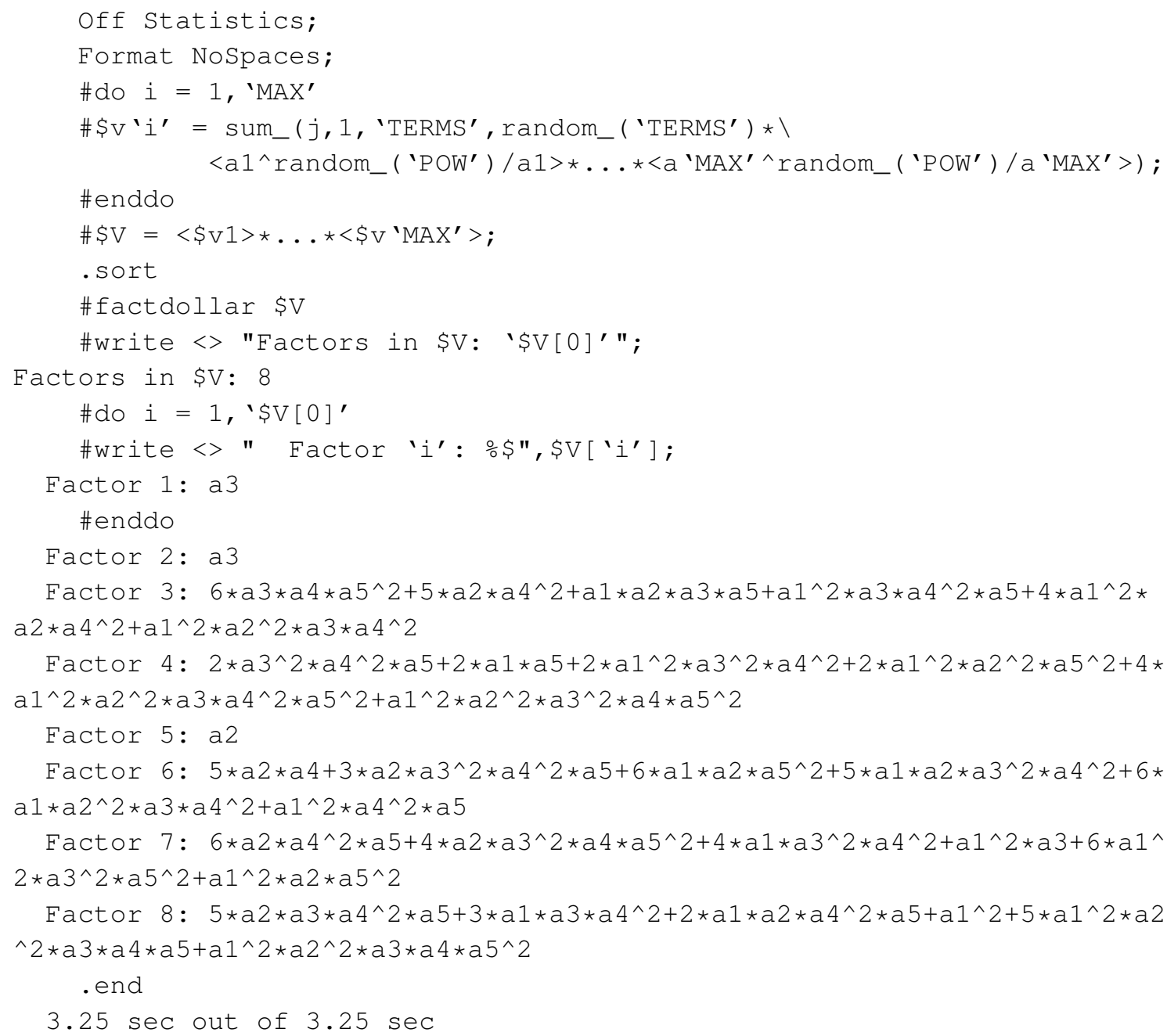

We refer to the factors as if they are array elements. The zero element tells the number of factors.

Of course \$-variables can be used in two ways: during compilation as shown above, and during execution:

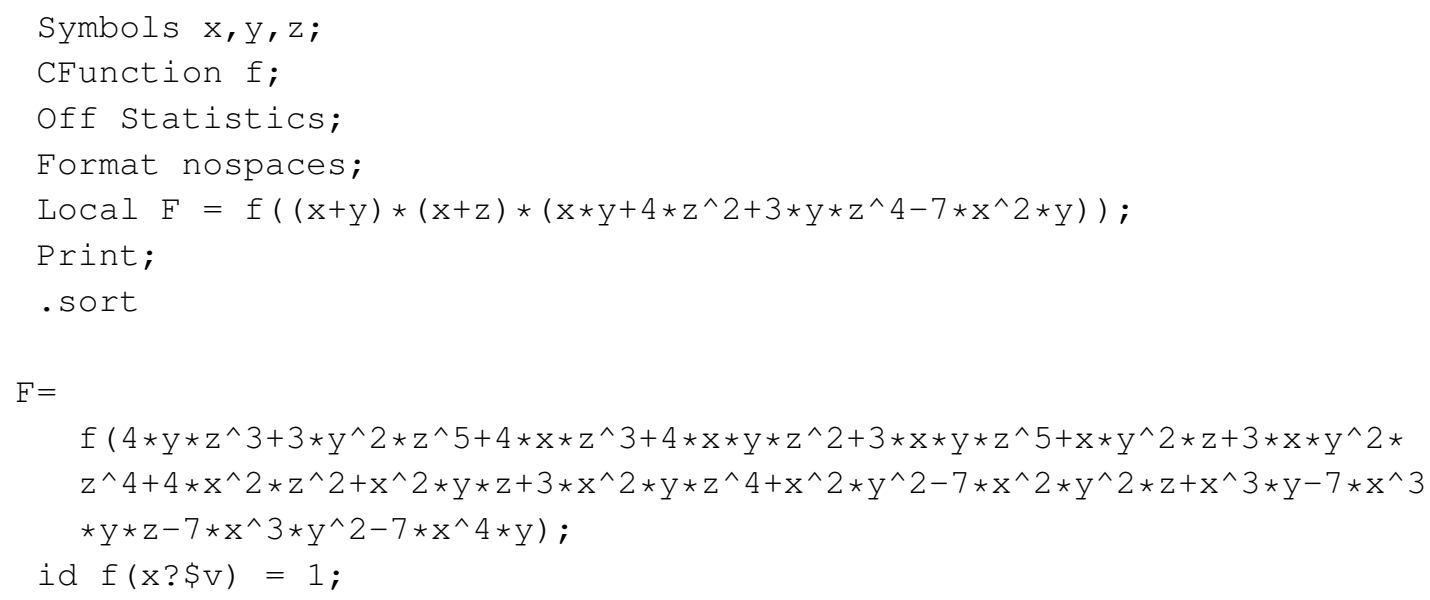




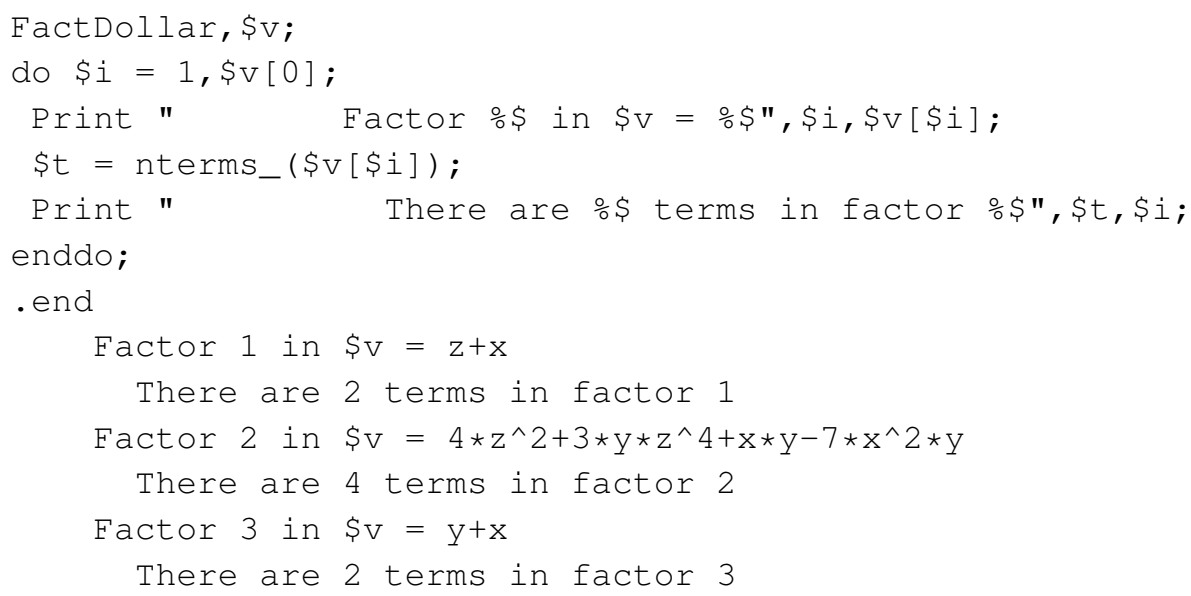

Here we need an extra supporting facility: the do loop during execution. Its variable is a \$-variable.

Internally the factorization algorithms work only with symbols and numbers. Yet we may use other objects as well. FORM will replace them temporarily by an internal set of symbols, called the "extra symbols". Then, after factorization these are replaced back. Hence the following example works properly.

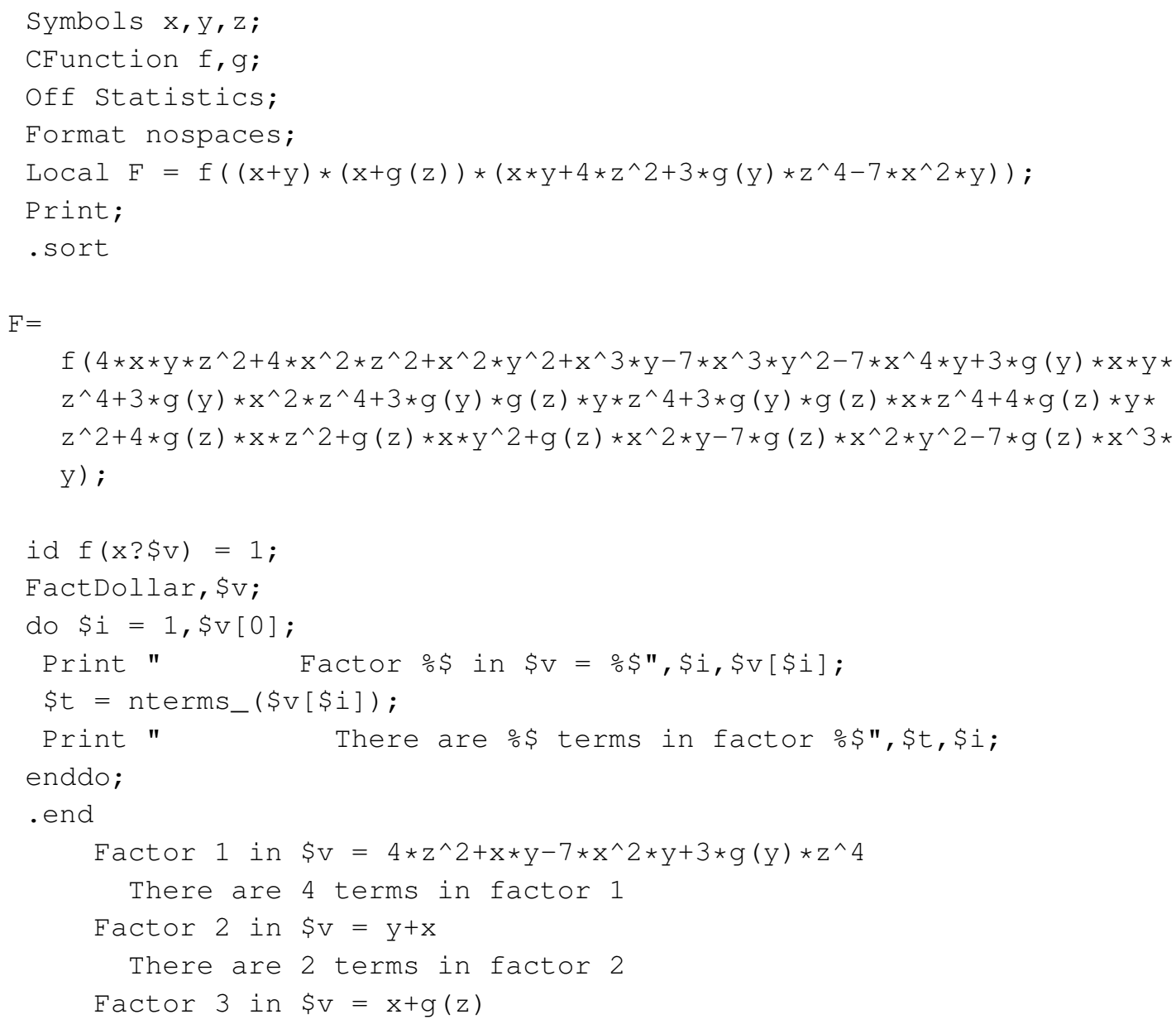


There are 2 terms in factor 3

There are more things that can be said about the factorization, but the talks is supposed to be finite in time.

\section{Rational Polynomials}

Another important thing that was missing, was the capability to deal with rational polynomials. This has even led to the introduction of the external channels to use other programs like FERMAT [3] for this purpose. It would have been nice to have FERMAT in the form of a library, like zlib (compression) or the GMP [4] (for multiprecision calculations), but that was not to be. Now we have our own capabilities.

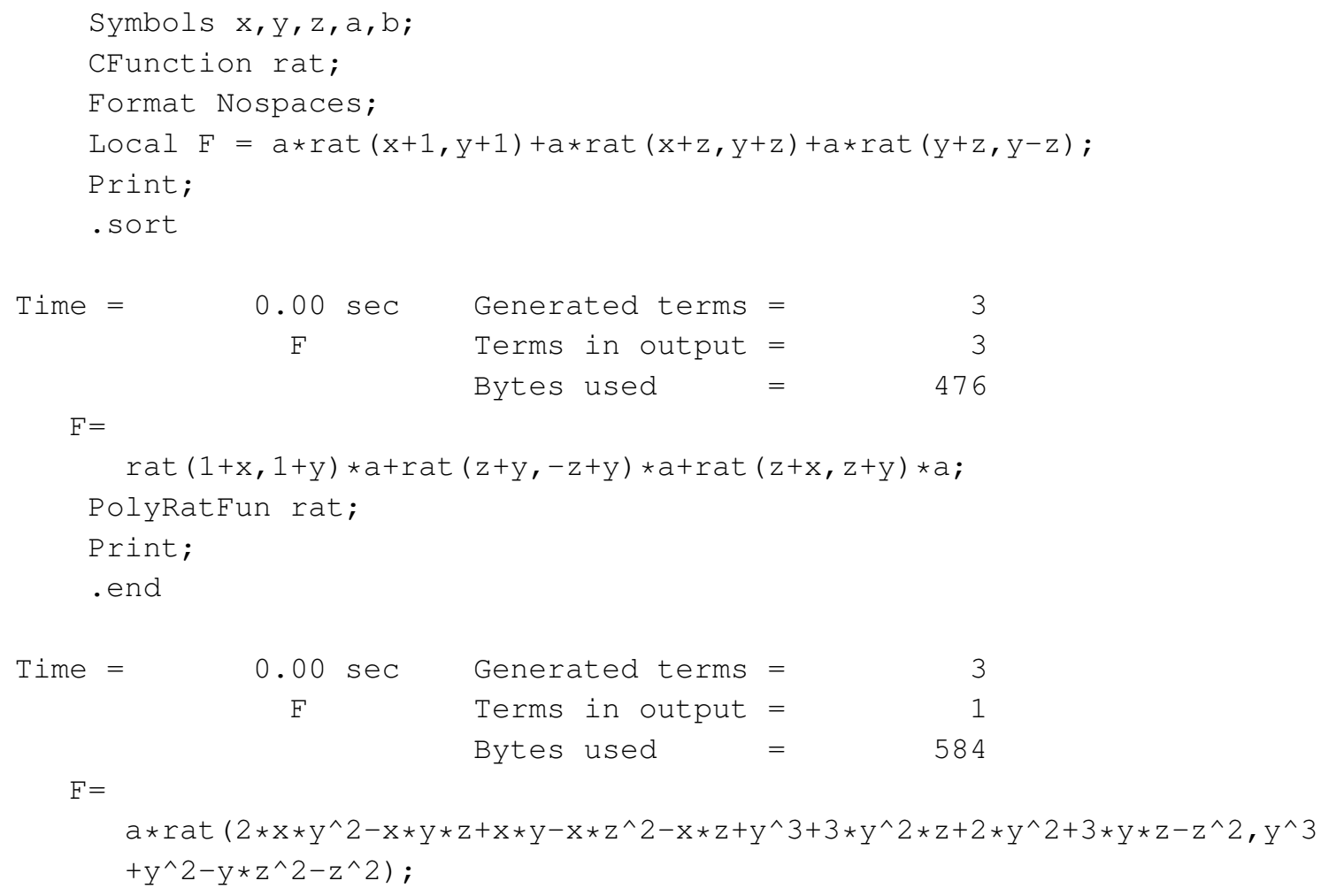

Like many things in FORM this is of course limited to the maximum size of the terms. If this turns out to be a limitation, there are usually other ways to attack the problem. In that case the numerators and denominators are very big expressions and it is better to store them in separate expressions or $\$$-variables. These then can be used in the new functions $\mathrm{gcd}_{-}$, div_, rem_to obtain results.

The first application of the rational polynomials was to make a new version of the Mincer [5] library. This version works exact. This means that it does not use expansions in $\varepsilon$. All $\varepsilon$ dependence is put inside the rational polynomial.

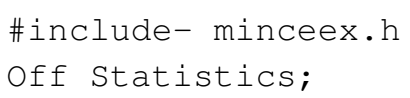




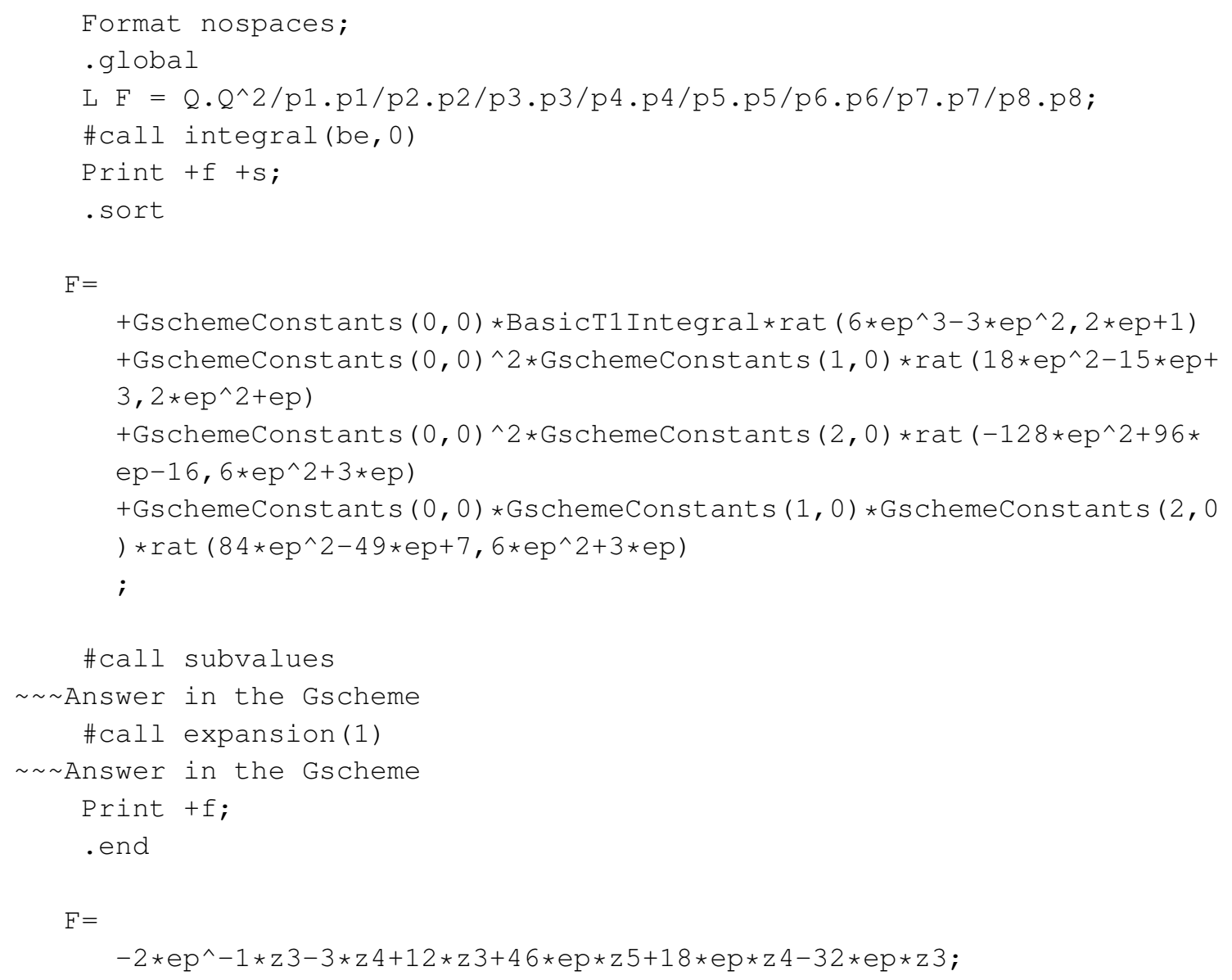

As is shown above, there are some constants, which are basic one loop integrals with zero, one or two insertions and there is a two loop integral of type $\mathrm{T} 1$ with one insertion. There is one more constant which is the basic non-planar integral in three loops.

The first three integrals are known in terms of $\Gamma$-functions and can be expanded as far as wanted.

The T1 integral can be expanded to any precision but that takes more and more time and runs eventually into the limitation that there are relations between the Multiple Zeta Values and these are known only to a certain weight [6]. Enough precision is built in for any practical calculations.

The NO integral is more of a problem, but is known to sufficient precision for even 4 loop calculations.

The above program shows that this exact treatment is quite good because we do not have to worry about cancellations of powers of $\varepsilon$.

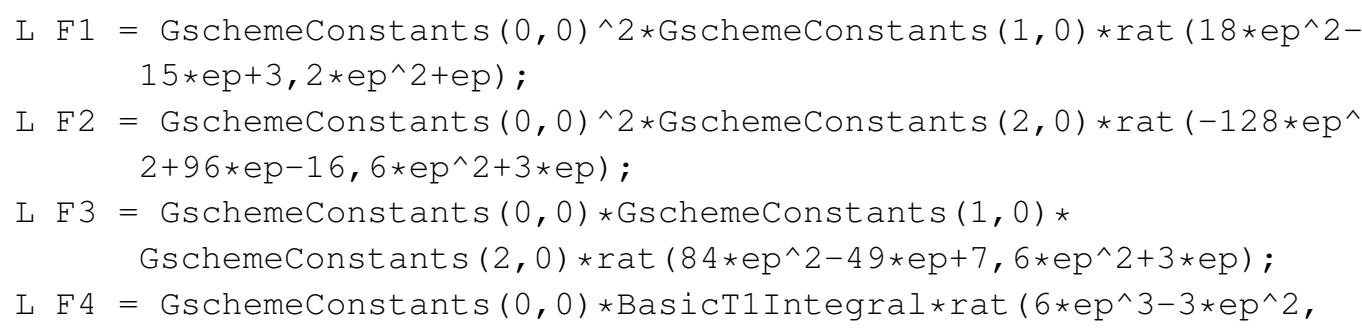


$2 \star e p+1)$;

\#call subvalues

$\sim \sim$ Answer in the Gscheme

\#call expansion(1)

$\sim \sim$ Answer in the Gscheme

Print $+f$;

. end

$\mathrm{F}=$

$-2 * e p^{\wedge}-1 * z 3-3 * z 4+12 * z 3+46 * e p * z 5+18 * e p * z 4-32 * e p * z 3 ;$

$\mathrm{F} 1=$

$192+3 * e p^{\wedge}-4-18 * e p^{\wedge}-3+48 * e p^{\wedge}-2-96 * e p^{\wedge}-1-18 * e p^{\wedge}-1 * z 3-27 * z 4+108 * z 3-$

$384 * e p-126 * e p * z 5+162 * e p * z 4-288 * e p * z 3$;

$\mathrm{F} 2=$

$-1024 / 3-16 / 3 * e p^{\wedge}-4+32 * e p^{\wedge}-3-256 / 3 * e p^{\wedge}-2+512 / 3 * e p^{\wedge}-1+256 / 3 * e p^{\wedge}-1 *$ $z 3+128 * z 4-512 * z 3+2048 / 3 * e p+1024 * e p * z 5-768 * e p * z 4+4096 / 3 * e p * z 3$;

$\mathrm{F} 3=$

$448 / 3+7 / 3 * e p^{\wedge}-4-14 * e p^{\wedge}-3+112 / 3 * e p^{\wedge}-2-224 / 3 * e p^{\wedge}-1-154 / 3 * e p^{\wedge}-1 * z 3-$

$77 * z 4+308 * z 3-896 / 3 * e p-546 * e p * z 5+462 * e p * z 4-2464 / 3 * e p * z 3$;

$\mathrm{F} 4=$

$-18 * e p^{\wedge}-1 * z 3-27 * z 4+108 * z 3-306 * e p * z 5+162 * e p * z 4-288 * e p * z 3 ;$

As one can see, there are quite a few terms cancelling between the terms with only one loop constants.

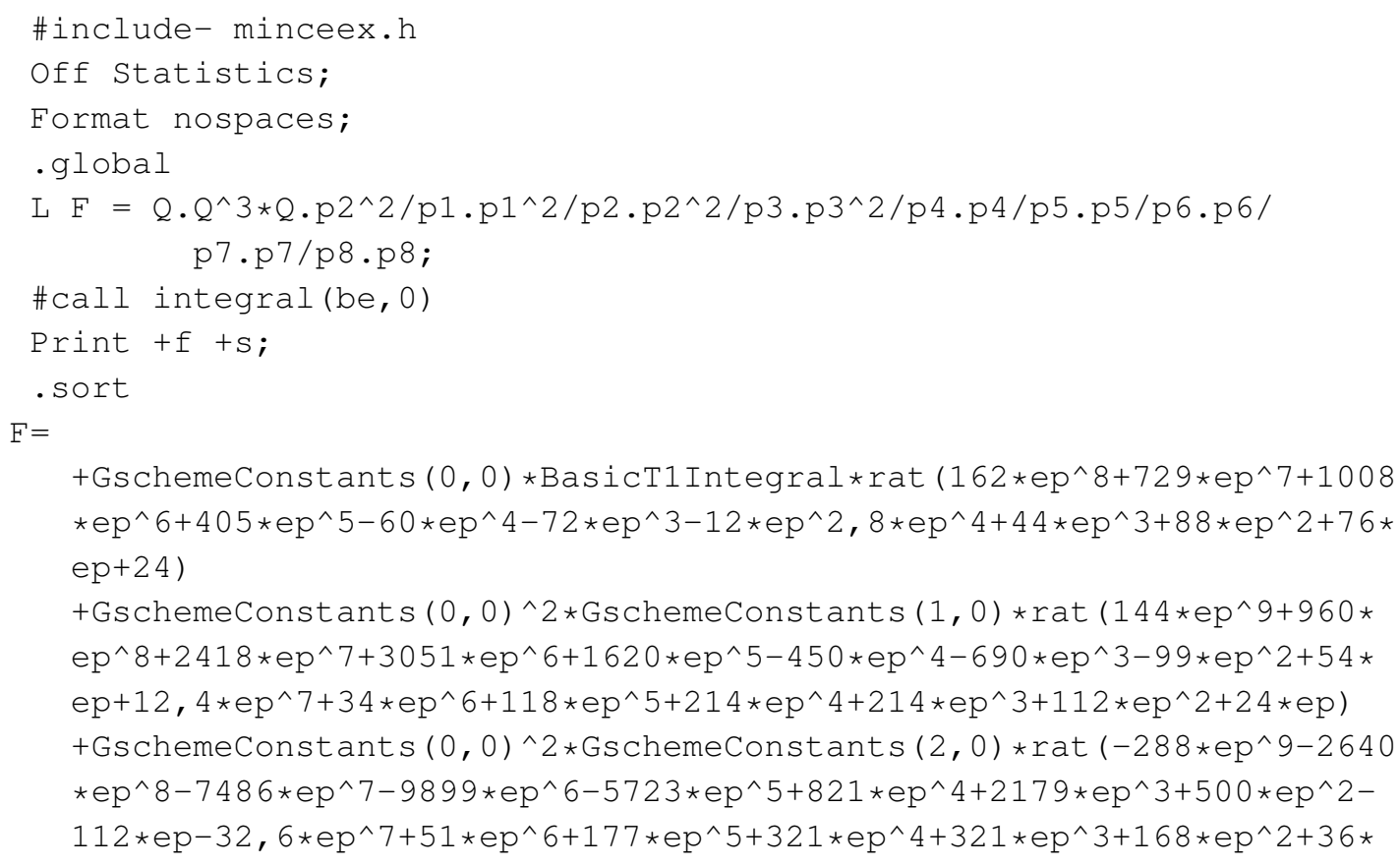




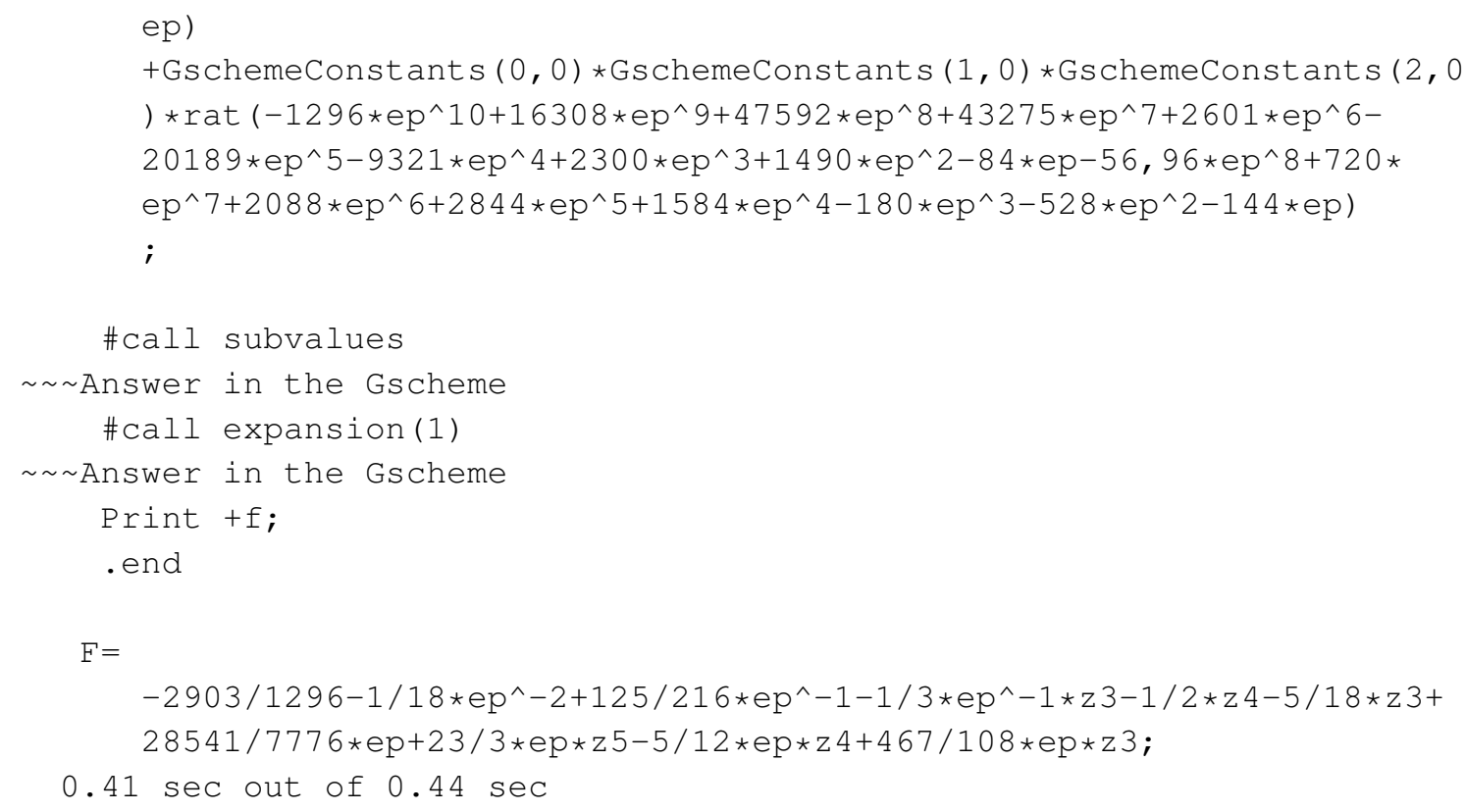

The "Mincer Exact" package has been added to the FORM distribution.

\section{New Functions}

FORM has obtained many new functions. We name them here. Some names are selfevident:

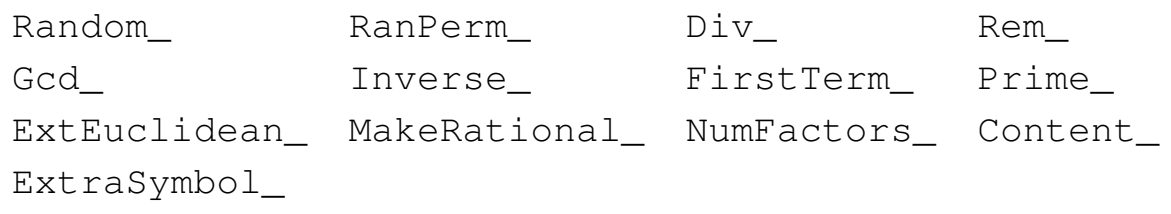

A number of these functions are designed for use in future packages, like a package for Gröbner bases. Such bases can often be calculated faster when calculus is over a prime number and in the end the results over several prime number calculations are combined into a result modulus the product of these numbers.

Making a decent Gröbner basis package is a major undertaking. Again the better packages are not suitable for inclusion as a library and are usually part of a commercial product. There is much heuristics involved to take shortcuts and all of that is kept secret. This means that one has to develop ones own heuristics. For this reason we have only been experimenting a little bit with Gröbner bases.

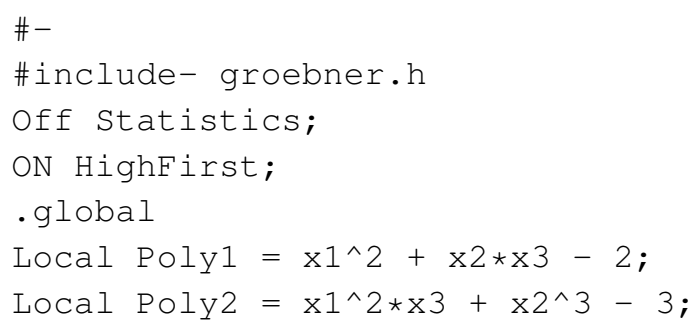




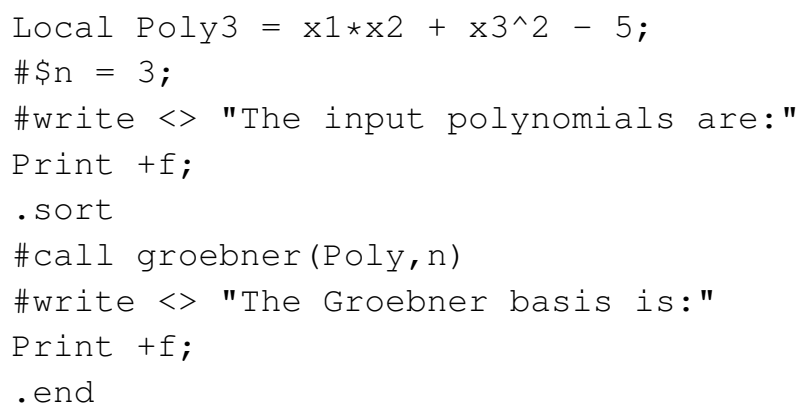

The above shows what this should look like from the users perspective. The result of this program is:

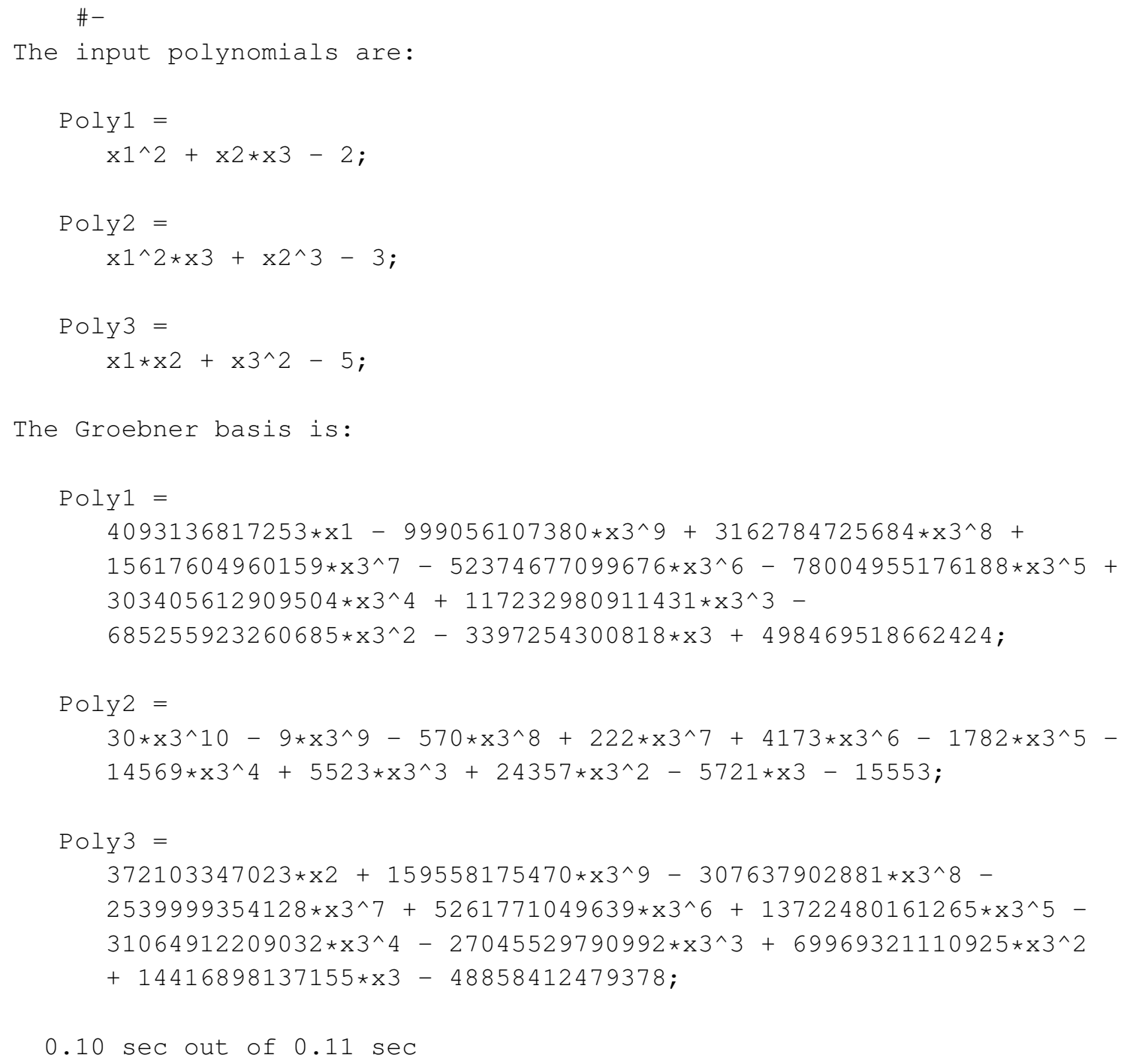

One can see here that this can run out of hand rather quickly. Of course the secret is in what is in the library groebner.h. It uses a large number of the new functions.

This is for example a routine that defines an S-polynomial: 


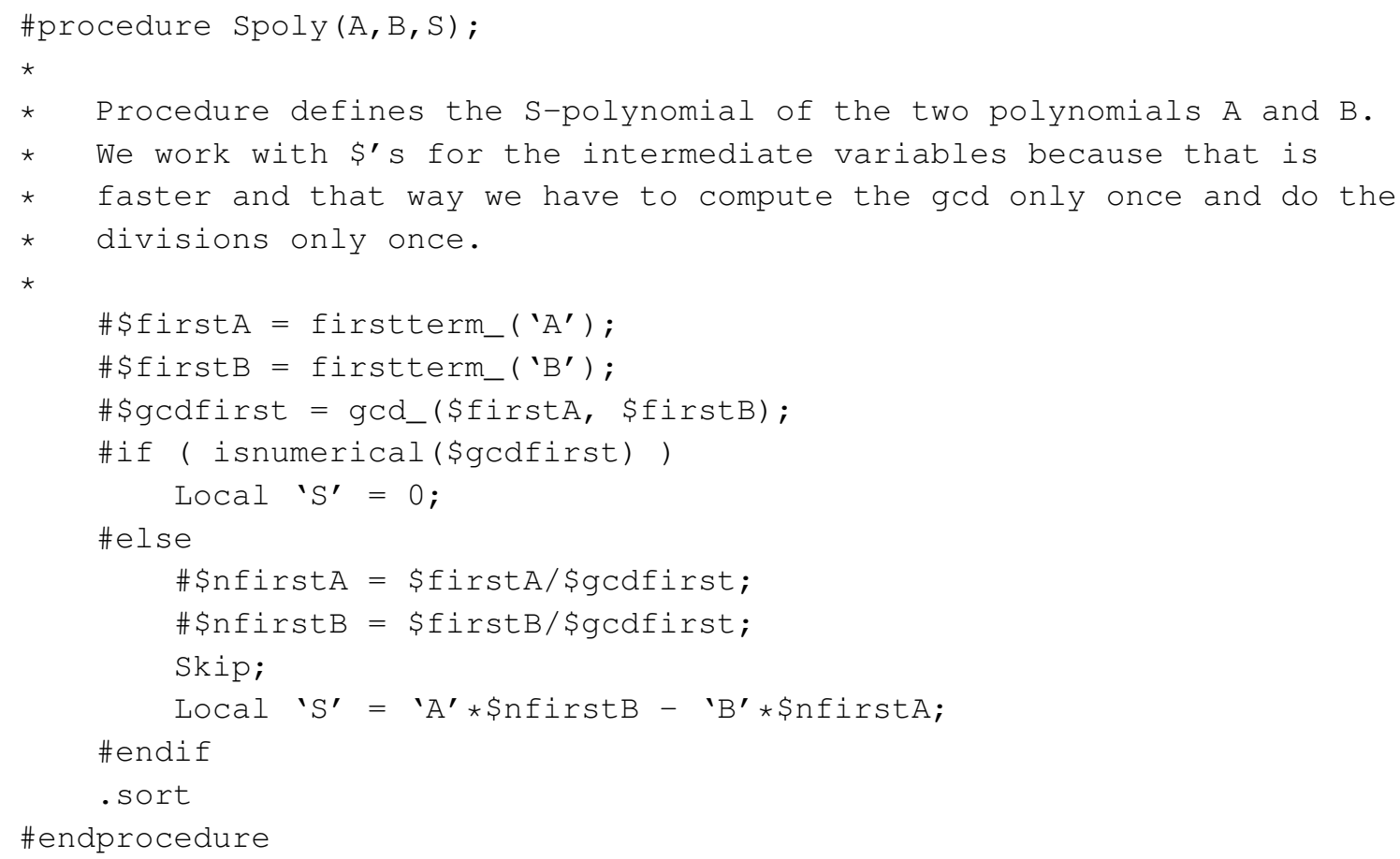

Note the use of the functions firstterm_and gcd_. Also the new option isnumerical in the preprocessor if statement is being used. Another routine:

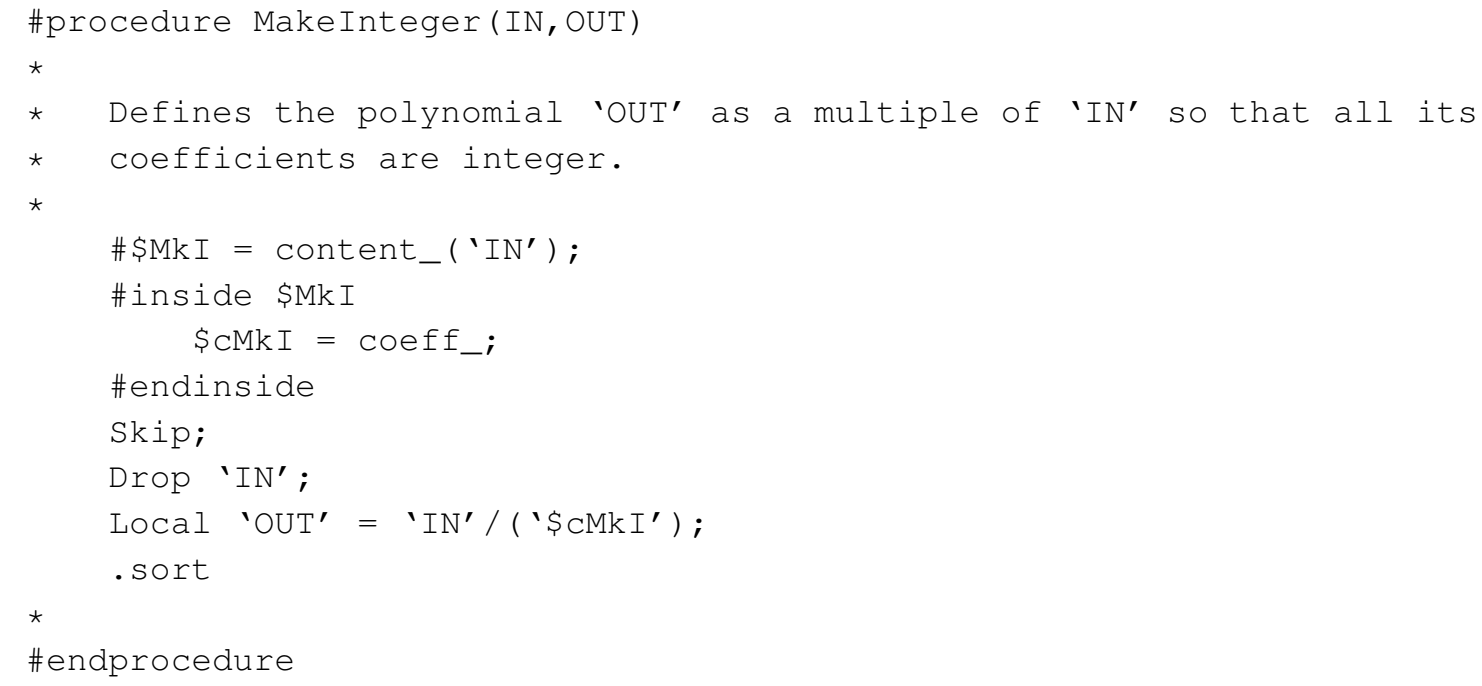

Here we use the function content_ to eventually obtain the GCD of the numerators and the LCM of the denominators.

Now we hope for volunteers to make a good package.

\section{Miscellaneous}

The parallel versions TFORM and ParFORM are both fully functional now. Till about a year ago ParFORM was still missing much of the functionality and was also not very portable. This has 
all been rectified. It is part of the open source distribution. It does however need a proper MPI installation.

One of the complaints in the past was that sav files of different executables of FORM were incompatible. This meant that a sav file generated on one computer might not be usable on another. Also new versions would often need extra variables in the .sav file and hence the old files would be useless. Starting with version 4.0 we have made an attempt to solve these problems. The files should now be uniform, even between 32-bits and 64-bits versions. In addition we have left much spare space in the headers to allow for future extensions that would otherwise invalidate old files.

Of course, some files cannot be carried from a 64-bits computer to a 32-bits computer. If we use $x^{123456}$, the power is more than the maximum power allowed for symbols on 32-bits systems. Similarly one can exceed the total number of different objects used in all expressions together. But those are rather natural limitations and they would occur only in exceptional cases.

Checkpoints are selected points in a FORM program from which the program can be restarted. One can define many such checkpoints but the program will remember only the last one it has encountered. This facility allows the user to restart the program when external causes have halted execution, like a power outage. The user has to define these checkpoints. It is not done automatically.

There is now a forum for FORM users to communicate with each other. To post on the forum one needs to register. This involves answering an easy question and then waiting till one of the moderators approves of the registration. This procedure is needed because of SPAM attacks and organizations having automatic programs for trying to register on forums like this.

The forum is the proper way to report bugs, to ask questions about installation and versions, or to ask help with certain features or techniques.

For all features in FORM, TFORM and ParFORM holds that we have tried our best to make them running flawlessly on systems that are available to us. Yet it is not excluded that on other systems strange things happen. This may be due to errors in the other systems, unanticipated behaviour, or just insufficiently careful programming on our side. Whenever such a thing occurs we ask the user to report the problems by means of the forum.

\section{What brings the future?}

The one line answer to this question is:

Hopefully something spectacular.

Currently we (mainly Jan) are trying to make a system for rewriting outputs for numerical programs in a way that takes as few operations as possible. For this we will be applying a completely new method. Hopefully there will be some results in the autumn. Already we have a first order program which is more classical and gives results similar to Haggies [9] and the external code that was made for the Grace system [10, 11]. This is not available yet because it needs extra supporting code and most likely it will change. But I have here one example:

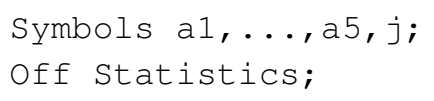




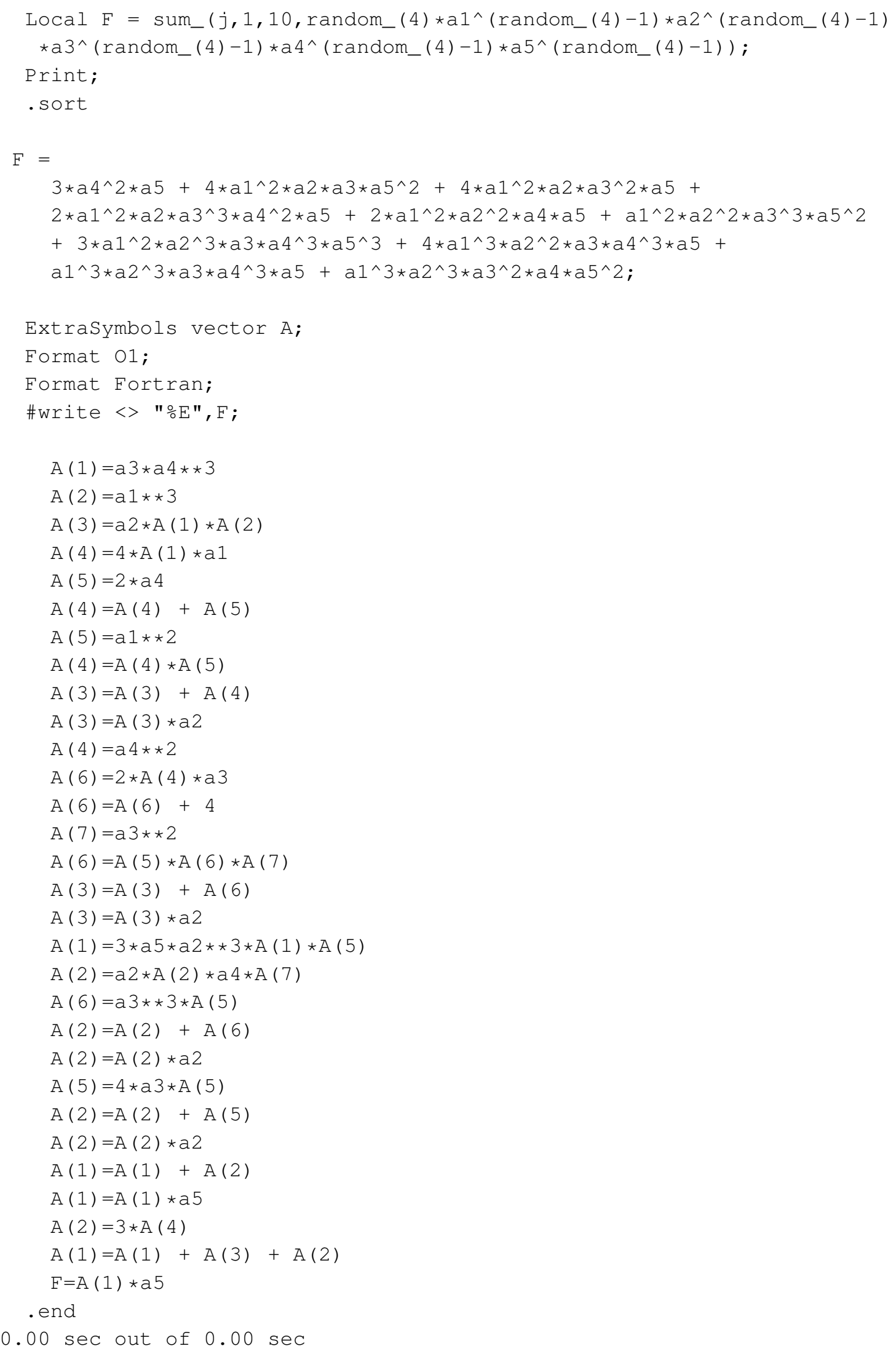


Of course this is not optimal. It is just a first order approximation.

\section{References}

[1] J. A. M. Vermaseren, New features of FORM, arXiv math-ph/0010025.

[2] M. Tentyukov and J. A. M. Vermaseren, The multithreaded version of FORM, Comput. Phys. Commun. 181 (2010) 1419, arXiv hep-ph/ 0702279.

[3] R.H. Lewis, Fermat, http://www.bway . net/ lewis/.

[4] GNU Multiple Precision Arithmetic Library, http://gmplib.org/.

[5] Mincer is a program for the evaluation of massless three loop propagator diagrams. The original paper is: S.G. Gorishnii, S.A. Larin, L.R. Surguladze and F.V. Tkachev, Comput. Phys. Commun. 55 (1989) 381-408. A much improved FORM version is included in the FORM distribution.

[6] J. Blumlein, D.J. Broadhurst and J.A.M. Vermaseren, Comput. Phys. Commun. 181 (2010) 582-625.

[7] J. Kuipers, J. A. M. Vermaseren, A. Plaat and H. J. van den Herik, Improving multi-variate Horner schemes with Monte Carlo tree search, arXiv 1207.7079.

[8] J. Kuipers and J. A. M. Vermaseren. Code simplification in FORM, In preparation.

[9] T. Reiter, Optimising code generation with haggies, Comput. Phys. Commun. 181 (2010) 1301-1331. arXiv 0907.3714.

[10] F. Yuasa et al., Automatic computation of cross sections in HEP, Prog. Theor. Phys. Suppl. 138 (2000) 18. arXiv hep-ph/0007053.

[11] J. Fujimoto et al., GRACE with FORM, Nucl. Phys. Proc. Suppl. 160 (2006) 150-154. 